\title{
Application of ANP-PCE in post evaluation of water conservancy project
}

\author{
Xinyi Chen ${ }^{1, *}$ \\ ${ }^{1}$ Management Science and engineering, School of management, Tianjin University of Technology, China
}

\begin{abstract}
Combined with the characteristics of post evaluation of water conservancy projects, this paper presents an integrated comprehensive evaluation method based on the combination of fuzzy comprehensive evaluation and network analysis, namely ANP-PCE method. Then the applicability and scientificity of the ANP-PCE method are verified by taking a water conservancy project in Shaanxi Province as an example. The research shows that: in the post evaluation of water conservancy project, the ANP-PCE method has strong accuracy and reliability, the evaluation method shows strong applicability, and has a certain promotion value.
\end{abstract}

\section{Introduction}

China built a large number of water conservancy facilities in the 1950s-1960s. However, due to the limitation of operation and maintenance level, management ability and construction technology, most of the water conservancy facilities are in disrepair and out of management. Therefore, the post evaluation of such built water conservancy projects is of great significance to improve the management level and the formulation of system and policy ${ }^{[1]}$. Based on the detailed analysis of ANP method and fuzzy comprehensive evaluation method, ANP-FCE is established Taking a water conservancy project in Shaanxi Province as an example, the model is used to solve and deal with the fuzzy problems existing in the evaluation index and evaluation process, so as to provide certain theoretical support and scientific basis for improving the scientificity and accuracy of the regional water conservancy project post evaluation ${ }^{[2]}$.

\section{ANP-PCE Method}

\subsection{Theoretical Methods}

The selection of comprehensive evaluation method and the determination of index weight are two important contents in the process of water conservancy project post evaluation. The ANP-FCE integrated comprehensive evaluation method is used to solve the weight of each evaluation index.

\subsection{ANP Method to Determine Weight}

ANP is to divide the evaluation objectives into several levels, and through the establishment of hierarchical structure model to deal with the indicators, it can solve the problem of multi criteria complex decision-making without structural characteristics ${ }^{[3]}$. In this paper, the analytic hierarchy process (AHP) is used to analyse the indicators of post evaluation. The calculation steps and methods are as follows

1) Establish ANP network structure. The criteria and constraints of the network layer are shown in Figure 1.

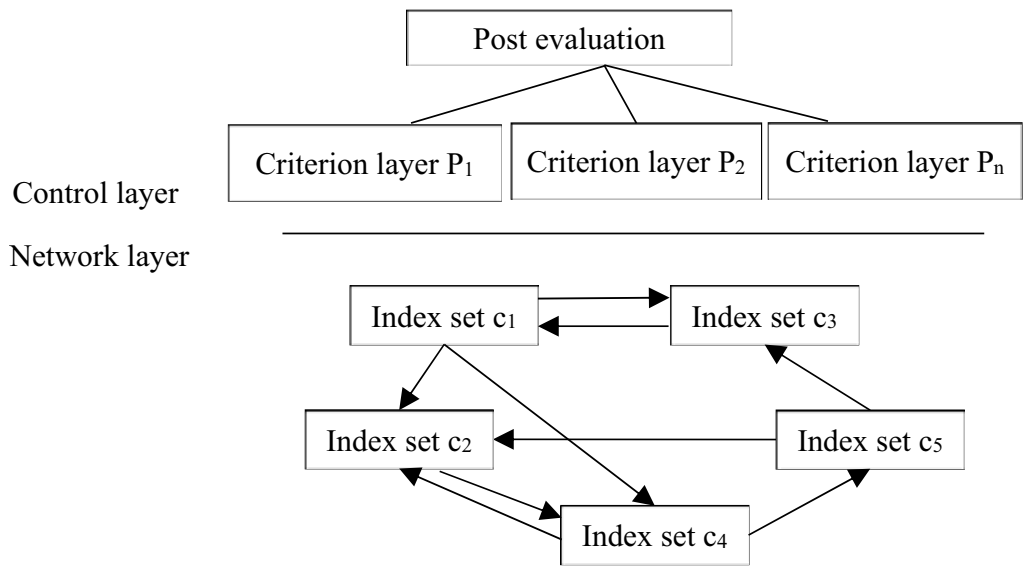

Fig. 1. ANP network structure diagram

\footnotetext{
*Corresponding author: 525245995@qq.com
} 
2) ANP discriminant matrix is constructed according to the criterion. Assuming that there are $\mathrm{m}$ post evaluation objectives or elements, each element is compared and analyzed in pairs according to the 1- 9 scale method, Among them, $\mathrm{a}_{\mathrm{ij}}$ represents the importance degree of $\mathrm{i}$ index relative to $\mathrm{j}$ index. Therefore, an m-order matrix can be constructed to determine the priority weight of each target under certain criteria. The matrix is the discriminant matrix, which is $A=\left(a_{i j m \times m}\right)$. It is assumed that $a_{i j} ;(i, j, k=$ $1,2, \ldots, \mathrm{m})$ can be estimated accurately, so we can get $\mathrm{a}_{\mathrm{ij}}=1 / \mathrm{a}_{\mathrm{ji}}, \mathrm{a}_{\mathrm{ij}}=\mathrm{a}_{\mathrm{ik}} \times \mathrm{a}_{\mathrm{kj}}, \mathrm{a}_{\mathrm{ij}}=1$, The discriminant matrix can be used to analyse the factors $\omega_{1}, \omega_{2}, \ldots$, $\omega_{\mathrm{n}}$ in the relative ranking weight under criterion A.

3) Use the single criterion condition to test the consistency of each factor. Assumed that RI is a random index, if consistency index $\mathrm{CI}$ satisfied $\mathrm{CI}=$ $\left(\lambda_{\max -m}\right) /(\mathrm{m}-1)$, Then the consistency ratio can be calculated by the following formula: $\mathrm{CR}=\mathrm{CI} / \mathrm{RI}$; If $\mathrm{CR}=0.1$, use the following formula to calculate the max-eigen: $\lambda_{\text {max }}^{\prime}=\mathrm{CI}(\mathrm{m}-1)+\mathrm{m}=0.1 \mathrm{RI}+(\mathrm{m}-$ 1) +1 ; If $n=1,2, C R=0$; The reason is that the first and second order discriminant matrices have the same consistency; If $\mathrm{n} \geq 3, \mathrm{CI}<0.1$, indicates that the consistency of the discriminant matrix is acceptable, otherwise the discriminant matrix should be revised again.

4) Use the general decision-making scheme for ranking. Use the top-down ranking method to calculate the importance weight vector of each element at the same level to the highest level; The weight vector of $n_{k-1}$ elements on $\mathrm{k}-1$ level in cascade structure relative to the total target is: $\omega(\mathrm{k}-1)=\left[\omega_{1}(\mathrm{k}-1), \omega_{2}(\mathrm{k}-1)\right.$, $\left.\omega_{\mathrm{n}}(\mathrm{k}-1)(\mathrm{k}-1)\right]^{\mathrm{T}}$. Finally, the weight vector of each level relative to the total goal and the upper level can be obtained.

It is complicated to solve the weight of each index according to the above process and formula, so this study uses SD software to process and analyze the index data of each level.

\subsection{Construction of ANP-FCE Evaluation Model}

1) We assumed that $u_{i}$ is the factor index of the i-th impact evaluation result, according to which the post evaluation index system can be established: $U=\left\{\mathrm{u}_{1}\right.$, $\left.\mathrm{u}_{2}, \ldots, \mathrm{u}_{\mathrm{n}}\right\}$.

2) Use $v_{j}$ to introduced as the $j$-th standard level of post impact assessment, Therefore, constructed the evaluation set: $\mathrm{V}=\left\{\mathrm{v}_{1}, \mathrm{v}_{2}, \ldots, \mathrm{v}_{\mathrm{m}}\right\}$. According to the actual situation of water conservancy projects, the corresponding evaluation grades $\mathrm{v}_{1}-\mathrm{v}_{5}$ are excellent, good, medium, poor and very poor.

3) Use single factor method to evaluate the membership grade of each evaluation index. By solving the membership degree $r_{i j}$ of qualitative and quantitative indexes, the judgment matrix R of water conservancy project post evaluation can be established as follows:

$$
\mathrm{R}=\left[\begin{array}{cccc}
r_{11} & r_{12} & \cdots & r_{1 m} \\
r_{12} & r_{22} & \cdots & r_{2 m} \\
\vdots & \vdots & \vdots & \vdots \\
r_{n 1} & r_{n 2} & \vdots & r_{n m}
\end{array}\right]
$$

4) Use ANP method to determine the weight of each evaluation index: $\mathrm{W}=\left(\mathrm{W}_{1}, \mathrm{~W}_{2}, \ldots, \mathrm{W}_{\mathrm{n}}\right)$. Among them, the importance degree of $u_{i}$ index to the evaluation result is $\mathrm{W}$, the value range is $0-1$, and the sum of each index is 1 .

5) The fuzzy operation of R and W based on the above results can obtain the comprehensive evaluation results. Further analysis of the post evaluation results of water conservancy projects can make an accurate judgment.

\section{Example Application}

\subsection{Construct the Evaluation Index and Comment Set}

In this paper, a water conservancy project in Shaanxi Province is selected as the research object. By selecting evaluation indexes and classifying them, a set of post evaluation index system can be formed, which can include all aspects of the project and fully reflect the actual working conditions ${ }^{[4]}$. According to this, this paper establishes 17 evaluation index systems including 3 evaluation levels from 4 aspects of water conservancy project construction process, as shown in Table 1.

Table 1. Post Evaluation Index System of Water Conservancy Project

\begin{tabular}{|c|c|c|}
\hline Target layer A & Criterion layer B & Network layer C \\
\hline \multirow{2}{*}{$\begin{array}{c}\text { Post evaluation } \\
\text { of water } \\
\text { conservancy } \\
\text { project } \mathrm{W}\end{array}$} & $\begin{array}{c}\text { Process evaluation } \\
\mathrm{B}_{1}\end{array}$ & $\mathrm{C}_{11}, \mathrm{C}_{12}, \mathrm{C}_{13}, \mathrm{C}_{14}, \mathrm{C}_{15}, \mathrm{C}_{16}$ \\
\cline { 2 - 3 } & $\begin{array}{c}\text { Economic benefit } \\
\text { evaluation } \mathrm{B}_{2}\end{array}$ & $\mathrm{C}_{21}, \mathrm{C}_{22}, \mathrm{C}_{23}, \mathrm{C}_{24}, \mathrm{C}_{25}$ \\
\cline { 2 - 3 } & $\mathrm{B}_{3}$ & $\mathrm{C}_{31}, \mathrm{C}_{32}, \mathrm{C}_{33}$ \\
\cline { 2 - 3 } & $\begin{array}{c}\text { Impact assessment } \\
\text { assessment } \mathrm{B}_{4}\end{array}$ & $\mathrm{C}_{41}, \mathrm{C}_{42}, \mathrm{C}_{43}$ \\
\hline
\end{tabular}

Combined with the actual situation of the project, the subsequent evaluation standards can be divided into five standard grades I-V: V $=\left\{\mathrm{v}_{1}, \mathrm{v}_{2}, \mathrm{v}_{3}, \mathrm{v}_{4}, \mathrm{v}_{5}\right\}$, and the corresponding comment sets are excellent, good, medium, poor and very poor.

\subsection{Determination of Weight}

For the index layer and criterion layer, the weight of each index is calculated as follows: $\mathrm{W}_{1}$ is $0.530,0.252,0.131$, 0.087 ; W2 is $0.031,0.038,0.123,0.056,0.197,0.066$, $0.068,0.041,0.026,0.041,0.020,0.080,0.034,0.045$, $0.014,0.027$. 


\subsection{Fuzzy Evaluation}

Membership function is used to represent the influence degree of each index. When the index is higher than the first level standard value, the membership degree corresponding to the excellent standard is 1 , while the membership degrees of other grades are 0 . When the evaluation index value is in the interval of two standard values, the corresponding membership degree can be calculated according to the following formula:

$$
\begin{gathered}
r_{i j}=1-r_{i}(j+1) \\
r_{i}(j+1)=\left(v_{i, j}-x_{i, j}\right) /\left(v_{i, j}-v_{i, j+1}\right)
\end{gathered}
$$

In the formula: $\mathrm{r}_{\mathrm{ij}}, \mathrm{r}_{\mathrm{i}(\mathrm{j}+1)}$ are the membership degrees of the $\mathrm{j}$ and $\mathrm{j}+1$ grades.

According to this, the membership matrix of quantitative index value of water conservancy project post evaluation is as follows:

$$
\mathrm{R}_{1}=\left[\begin{array}{llllll}
0.0 & 1.0 & 1.0 & 1.0 & 0.0 & 1.0 \\
0.8 & 0.0 & 0.0 & 0.0 & 0.0 & 0.0 \\
0.2 & 0.0 & 0.0 & 0.0 & 0.6 & 0.0 \\
0.0 & 0.0 & 0.0 & 0.0 & 0.4 & 0.0 \\
0.0 & 0.0 & 0.0 & 0.0 & 0.0 & 0.0
\end{array}\right]
$$

By inviting experts who are familiar with the general situation of the project to organize an evaluation team and carry out post evaluation on the project, the score is made according to the evaluation grade standard and the value range of the assigned value. The results are shown in Table 2 .

Table 2. Formatting sections, subsections and subsubsections.

\begin{tabular}{c|c|c|c|c|c|c|c|c|c|c}
\hline Evaluating indicator & \multicolumn{7}{|c}{ Expert points } \\
\hline Project decision & 5 & 4 & 5 & 5 & 4 & 4 & 3 & 5 & 4 & 3 \\
\hline Planning and design & 5 & 3 & 4 & 4 & 5 & 4 & 5 & 4 & 4 & 2 \\
\hline Construct & 3 & 5 & 5 & 3 & 4 & 5 & 3 & 4 & 5 & 4 \\
\hline Management & 4 & 3 & 3 & 3 & 3 & 2 & 4 & 3 & 3 & 5 \\
\hline Security & 4 & 4 & 4 & 4 & 3 & 5 & 5 & 4 & 4 & 5 \\
\hline Arrangement & 5 & 2 & 5 & 2 & 2 & 3 & 3 & 5 & 5 & 2 \\
\hline Social influence & 2 & 5 & 2 & 5 & 4 & 4 & 4 & 2 & 3 & 4 \\
\hline Environmental effect & 5 & 4 & 4 & 4 & 2 & 4 & 5 & 4 & 5 & 5 \\
\hline Sustainability & 3 & 4 & 5 & 3 & 3 & 3 & 3 & 3 & 4 & 3 \\
\hline System and organization setup & 4 & 2 & 3 & 3 & 4 & 5 & 3 & 5 & 4 & 4 \\
\hline Infrastructure and policy & 5 & 5 & 4 & 2 & 4 & 5 & 5 & 4 & 3 & 3 \\
\hline
\end{tabular}

The membership degree of qualitative indicators is calculated by using relevant data and scoring rules, and the results are as follows:

$$
\mathrm{R}_{2}=\left[\begin{array}{llllllllll}
0.8 & 0.7 & 0.0 & 0.8 & 0.7 & 0.0 & 0.0 & 0.9 & 0.6 & 0.7 \\
0.3 & 0.2 & 0.3 & 0.1 & 0.3 & 0.3 & 0.2 & 0.1 & 0.4 & 0.3 \\
0.2 & 0.0 & 0.5 & 0.0 & 0.0 & 0.7 & 0.8 & 0.0 & 0.0 & 0.0 \\
0.0 & 0.0 & 0.2 & 0.1 & 0.0 & 0.0 & 0.0 & 0.0 & 0.0 & 0.0 \\
0.0 & 0.0 & 0.0 & 0.0 & 0.0 & 0.0 & 0.0 & 0.0 & 0.0 & 0.0
\end{array}\right]
$$

The comprehensive evaluation grade result of each index is: $\mathrm{V}=\mathrm{W}_{2} \mathrm{R}=[0.480,0.272,0.162,0.087,0]$. Therefore, it is judged that the evaluation level of the project is at level 1, that is, the project is in the optimal state.

\section{4 conclusion}

Although the progress of agricultural irrigation facilities is relatively slow, the benefit of agricultural irrigation is not obvious $^{[5]}$. The post evaluation of the project is an excellent project as a whole. The evaluation method used in this paper has strong applicability and reliability, and the evaluation results can better reflect the actual situation of water conservancy projects, with good consistency and reliability.

\section{References}

1. Xiaoguang Wang. Current situation, problems and suggestions of rural water conservancy projects in Fushun County. Application technology of soil and 
water conservation, 2 (2013)

2. Keshan Peng. Study on the problems of ecoenvironmental disasters in the Three Gorges area and the ways to reduce them. Application technology of soil and water conservation, 2 (1995)

3. Li Jiang, Youkun Wu. Study on the impact index of soil and water loss of water conservancy project. Application technology of soil and water conservation, 2 (2016)

4. Chenghong Liu. Study on ecological protection technology of water conservancy slope. Application technology of soil and water conservation, 2 (2010)

5. Xinxin Yang. Discussion on archives management of water conservancy projects. Water Conservancy Technical Supervision, 1 (2013) 